\title{
A Permanent solution to Restore Occlusal Vertical Dimension and Partial Edentulism - Overlay Removable Partial Denture
}

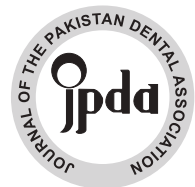

\author{
Surhan Aziz ${ }^{1}$ \\ BDS \\ Muhammad Rizwan Nazeer ${ }^{2}$ \\ BDS, FCPS \\ Kamil Zafar \\ BDS \\ Robia Ghafoor ${ }^{4}$ \\ BDS, FCPS
}

Tooth wear is a common problem seen in population and poses restorative challenge to a dentist as well. Extensive dental procedures are usually required for the treatment of tooth wear associated with loss of occlusal vertical dimension. An overlay removable partial denture provides conservative treatment option for patients with moderate to severe worn down dentition and partial edentulism. It is designed in such a way that some part of denture component also covers the occlusal surface of remaining teeth and replace the remaining teeth to achieve a functionally stable occlusion. This case report is about a patient who was given cast metal overlay upper and lower denture for a correction of lost vertical dimension of occlusion, replacement of few missing teeth and for the prevention from further tooth wear.

KEYWORDS: Tooth wear, vertical dimension, overlay denture

HOW TO CITE: Aziz S, Nazeer MR, Zafar K, Ghafoor R. A Permanent solution to restore occlusal vertical dimension and partial edentulism - Overlay removable partial denture. J Pak Dent Assoc 2021;30(1):66-69.

DOI: https://doi.org/10.25301/JPDA.301.66

Received: 10 June 2020, Accepted: 24 August 2020

$\mathrm{T}$ ooth wear is defined as the progressive loss of tooth structure from attrition, abrasion, erosion or abfraction. ${ }^{1}$ It has a multifactorial cause and can be localized to anterior or posterior teeth or often generalized throughout the dentition. ${ }^{2}$ When the frequency of tooth wear is slow, the resultant occluso-vertical dimension is often maintained through dento-alveolar compensation. ${ }^{3}$ However, the cases of accelerated tooth wear often results in loss of occluso-vertical dimension and hence an increase in freeway space is usually found.$^{3-4}$ Loss of occlusal vertical dimension (OVD) significantly affects patients quality of life by altering aesthetics and function, hence requires a systematic approach for appropriate management. ${ }^{5,6}$

The management of accelerated tooth wear is often complicated by patient complains (e.g pain and sensitivity), which requires immediate treatment to relief symptoms.

1. Post Graduate trainee Department of Operative Dentistry at the Aga Khan University Hospital, Karachi, Pakistan

2. Senior Registrar Department of Operative Dentistry at the Baharia University Medical and Dental College, Karachi, Pakistan.

3. Instructor, Department of Operative Dentistry at the Aga Khan University Hospital, Karachi, Pakistan

4. Assistant Professor, Department of Operative Dentistry at the Aga Khan University Hospital, Karachi, Pakistan.

Corresponding author: "Dr. Robia Ghafoor" < robia.ghafoor@aku.edu >
Following that, a preventive approach is employed for preventing further tooth loss. ${ }^{6}$ Definitive treatment plan is then aimed at regaining the lost occlusal vertical dimension, with fixed prosthodontics being the most preferred option. ${ }^{7}$ Extensive dental procedures such as multiple endodontics, post core, crown lengthening surgeries are usually required before fixed prosthodontics, which not only results in irreversible loss of pulp vitality but also a great deal of financial expenditure and time to achieve desire results., ${ }^{4,5,7,8}$

An overlay removable partial denture is a conservative treatment option for patients with moderate to severe worn down dentition and partial edentulism. ${ }^{9}$ It is designed in such a way that some part of denture component also covers the occlusal surface of remaining teeth and replace the missing teeth to achieve a functionally stable occlusion. ${ }^{9,10}$ It can also be used as transitional prostheses prior to fixed full mouth rehabilitations or in patients with severe skeletal deficiency such as ectodermal dysplasia, Kenny-Caffey Syndrome 11 and malocclusion, but can be considered as or as permanent prosthesis in severe tooth wear cases and in patient with financial and/or time constraints. ${ }^{4,12}$ The present case report is about a patient who was given cast metal overlay bimaxillary dentures for a correction of lost vertical dimension of occlusion, for replacement of few 
missing teeth and for the prevention from further tooth wear.

\section{CASE PRESENTATION}

A 53 year old male presented to the dental clinics of Aga Khan University Hospital, Karachi Pakistan with the complaint of extreme sensitivity in lower left jaw for several months. Pain aggravated on taking hot and cold beverages but since the last one week it also increases on taking room temperature water and lingers for more than few minutes after removal of the stimulus. The patient's medical history was insignificant, except hypertension for which he was on medications. His last visit to a dentist was 3 to 4 years ago for extraction of teeth on right side.

On extra oral examination, no swelling or lesions were present and findings for lymphadenopathy were negative. Muscles of mastication were tender to palpation and TMJ examination revealed no clicking or crepitus. Range of mandibular movements was within normal limits and freeway space was found to be $6 \mathrm{~mm}$ with reduced occlusal vertical dimension (OVD). On intraoral examination, soft tissues were normal. Teeth \# 17,16,47,46 were absent and generalized moderate to severe tooth wear was found. Increased overjet and overbite were also observed. Radiographs and pulp vitality test was performed for left lower teeth which revealed irreversible pulpitis in tooth \# 37 and reversible pulpitis in \# 36. Extra- and intra-oral photographs were initially taken for case assessment. (Figure $1 \& 2$ ) Questions regarding stress, lifestyle, diet, medications, parafunction, brushing habits frequency of acidic drinks were also inquired. Para-

Figure 1: Pre-operative intra-oral pictures

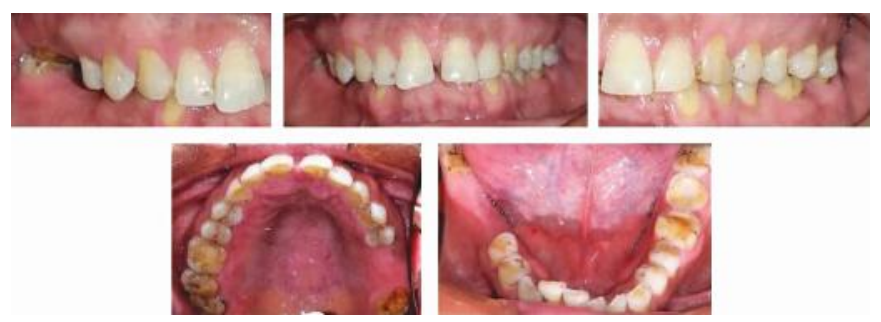

Figure 2: Pre-operative OPG Xray

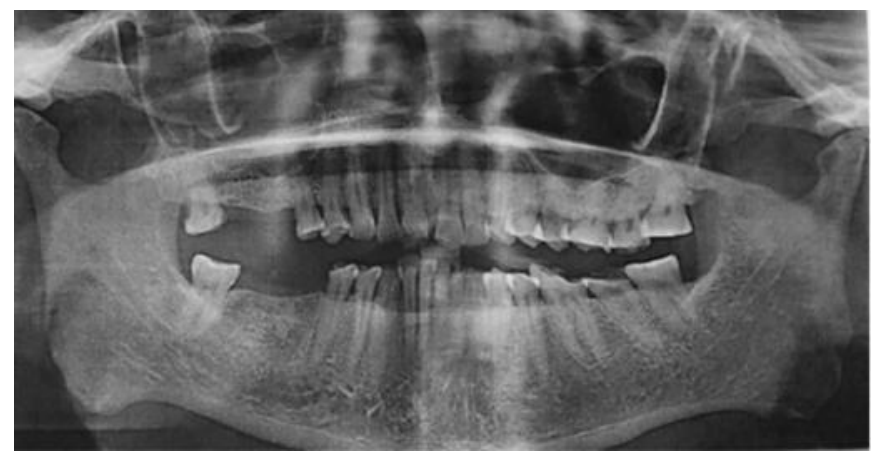

functional habit (clenching) was positive in our patient. Study casts were also obtained.

On the basis of thorough clinical and radiographical examination, problem list was consisted of:

1) Moderate to severe tooth wear with reduced occlusal vertical dimension of occlusion (freeway space $=6 \mathrm{~mm}$ ) without dentoalveolar compensation.

2) Irreversible puplitis \# 37

3) Reversible pulpitis \# 36

4) Missing teeth \# 16, 17, 46, 47.

The treatment plan comprised of:

Immediate management: Root canal treatment \# 37 and composite resin restoration \# 36 for the relief of pain and sensitivity. Patient was also provided stabilization splint to prevent further wear and establish new OVD.

Control phase: Topical fluoride and oral hygiene instructions were given and after 3 months patient was evaluated for definitive management.

Definitive phase: Placement of indirect full coverage restorations on all posterior teeth at an increased occlusal height, implant retained crown for missing teeth and metal palatal veneers on maxillary anterior teeth vs placement of an overlay partial denture.

Monitoring: Regular follow up were advised to evaluate the timely status of tooth wear.

Initially, emergency treatment of sensitivity was carried out which included endodontic treatment in tooth \# 37 for relief of patients symptoms and canals were obturated upto length. Composite restoration (3M Filtek P-60, 3M ESPE) was then placed in \# 36. Impressions were obtained with irreversible hydrocolloid and patient was provided stabilization split which served two purposes. One was to prevent further progression of tooth wear and other was to establish new OVD for the accommodation of definitive prosthesis. All treatment options regarding prosthetic rehabilitation and pros and cons of each was thoroughly discussed with the patient. Patient was asked to think over all the options before selecting the final treatment option. A daily application of sodium fluoride $1.1 \%$ gel was advised for management of generalized sensitivity.

At the later appointment, splint was adjusted and delivered to the patient. All treatment options were re-discussed. Since the patient had financial constraints and wanted the treatment in a shorter time period, hence opted for overlay removable partial denture. After 3 months mouth preparation was done and impression was obtained with aqusail soft putty regular set (Dentsply Middle east and Africa) for the fabrication of 
overlay removable partial denture. At the subsequent appointment, framework trial (with acrylic teeth replacing was performed) was carried out. The framework was passive fit and comfortable to the patient. Wax bite was taken with Rigisel 2X VPS bite registration material (Dentsply Middle east and Africa) directly from teeth and mounted on semi adjustable articulator (Hanau Articulator, Teledyne Hanau Buffalo, NY, USA) in the subsequent appointment. Patient's feedback was obtained regarding increase in occlusal vertical dimension. After patients approval the denture was finally processed in acrylic.

The final denture was delivered and occlusion was checked (Figure 3). Reorganized occlusion was successfully achieved as planned. Post-operative instructions regarding

Figure 3: Post-operative pictures

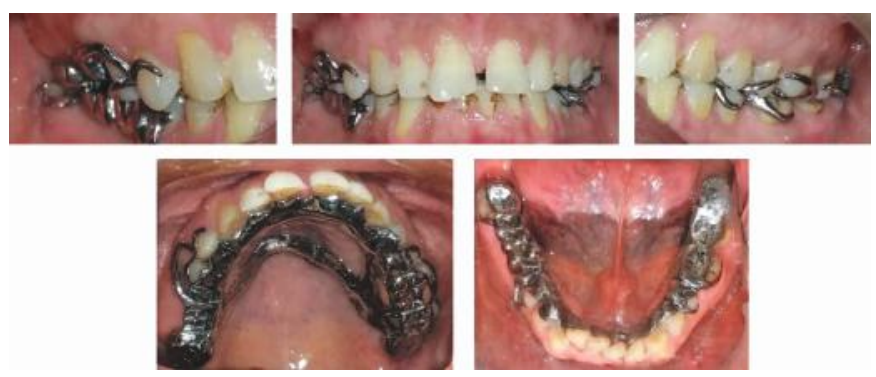

denture wearing, hygiene and food intake was given to the patient. Patient was asked to follow up after 24 hours. Mild discomfort was reported by the patient so muscle relaxants and analgesics were given for next three days. On a follow up visit after a week patient was doing absolutely fine with no discomfort. Patient was asked to wear prosthesis initially for all the time even during the sleep. After the patient got accustomed to the prosthesis, he was advised to wear the same for day time only. Next follow up was planned after 6 months.

\section{DISCUSSION}

The multifactorial etiology of tooth wear demands thorough history, clinical and radiographical examination together with special tests (TMJ screening, vertical dimension of occlusion, vitality tests) and occlusal cast examination before reaching the final diagnosis. ${ }^{10,11}$ Such patients usually presents with pain and sensitivity at advance wear stage, making the pathologic condition more complex. ${ }^{7}$ Those presenting with lost occluso-vertical dimension and partial edentulism often poses a great restorative challenge to a dentist. ${ }^{7,12}$ The management plan should be aimed at relieving acute patients complains, preventing further tooth wear, definitive prosthetic management which should be in the best interests of the patients and frequent monitoring throughout the treatment phase and afterwards. ${ }^{5,13}$ The treatment should be conservative, cost effective, comfortable and aesthetically pleasing to the patient. ${ }^{14,15}$

The initial management could vary depending on the clinical presentation such as from simple smoothening of a sharp edges of teeth, managing sensitivity by applying desensitizing agent or glass ionomer cement over exposed dentine. ${ }^{14}$ In cases with pulp exposure root canal treatment or dental extraction may be required in cases with severe tooth wear. ${ }^{14}$ We also followed a systematic approach in our patient, carried out endodontic treatment for relief of acute pain and also covered the exposed dentinal tubules with composite resin restoration for management of acute sensitivity in lower first molar. We also advised fluoride gel for management of generalized sensitivity and once the acute complained were settled, definitive prosthetic phase was initiated.

Depending upon the extent of tooth wear and associated loss of vertical dimension of occlusion, a patient can be categorized and are managed accordingly. ${ }^{7}$ Our patient had loss of occlusal vertical dimension with moderate tooth wear and wanted the most conservative, reversible and cost effective option. Therefore we planned an overlay denture because a single overlay denture can address multiple situations at a time such as it covers occlusal surfaces of teeth hence increase OVD, also protects teeth from further wear and sensitivity and can also replace missing teeth and porcelain veneer can also be applied on the metal framework covering anterior teeth. ${ }^{8-12,15-17}$ The other benefit of overlay denture is preservation of alveolar bone, as opposed to complete denture, therefore provides proprioception in patients with para-functional habits. ${ }^{15,16}$ Since the tooth wear was extensive in posterior teeth therefore the overlay denture covered the occlusal surface of all posterior teeth, replace the missing teeth and covered only the palatal surface of anterior teeth.

Despite multiple advantages of an overlay dentures, there are few disadvantages like compromised esthetics encountered by patients on removal of the prosthesis, increase food impaction and difficulty in maintaining oral hygiene which may lead to increased dental issues like caries and periodontitis. ${ }^{17}$ Since the overlay denture was not covering the anterior teeth in our patient hence the final outcome was aesthetically pleasing and comfortable to the patient.

It is essential for the management of tooth wear that emphasis should be given to regular recall and maintenance visits to ensure the long term success of overlay partial dentures. ${ }^{4}$ We also did periodic follow-ups after delivery of final prosthesis on day one, after a week and a month. Next follow-up is planned after six months. 


\section{CONCLUSION}

The purpose of the present case is to highlight the use of overlay removable partial dentures (ORPD) for the correction of lost occlusal vertical dimension (VDO) in a patient with moderately worn dentition, and partial edentulism. It is a conservative treatment option in cases with low esthetic concerns, if patients wish for simple procedures, limited finances or certain debilitating medical limitations.

\section{CONFLICT OF INTEREST}

None declared

\section{REFERENCES}

1. Donachie MA, Walls AW. The tooth wear index: a flawed epidemiological tool in an ageing population group. Community Dent Oral Epidemiol. 1996; 24:152-8.

https://doi.org/10.1111/j.1600-0528.1996.tb00833.x

2. Faigenblum M. Removable prostheses. Brit Dent J. 1999;186: 273-76

https://doi.org/10.1038/sj.bdj.4800086

3. Zengingul A, Eskimez S, Deger Y, Kama J. Tooth wears and dentoalveolar compensation of vertical height. Biotechnol Equip. 2007;21:362-65.

https://doi.org/10.1080/13102818.2007.10817474

4. Patel MB, Bencharit S. A treatment protocol for restoring occlusal vertical dimension using an overlay removable partial denture as an alternative to extensive fixed restorations: a clinical report. Open Dent J. 2009;3:213-18

https://doi.org/10.2174/1874210600903010213

5. Smith BG, Bartlett DW, Robb ND. The prevalence, etiology and management of tooth wear in the United Kingdom. J Prosthet Dent. 1997;78:367-72.

https://doi.org/10.1016/S0022-3913(97)70043-X

6. Bartlett DW. The role of erosion in tooth wear: aetiology, prevention and management. Int Dent J. 2005;55:277-84.

https://doi.org/10.1111/j.1875-595X.2005.tb00065.x

7. Turner KA, Missirlian DM. Restoration of the extremely worn dentition. J Prosthet Dent. 1984;52:467-74. https://doi.org/10.1016/0022-3913(84)90326-3
8. Windchy AM, Morris JC. An alternative treatment with the overlay removable partial denture: a clinical report. J Prosthet Dent. 1998; 79:249-53.

https://doi.org/10.1016/S0022-3913(98)70232-X

9. Beyth N, Tamari I, Buller Sharon A. Overlay removable denture for treatment of worn teeth. Spec Care Dentist. 2014; 34:295-97. https://doi.org/10.1111/scd.12063

10. Bataglion C, Hotta TH, Matsumoto W, Ruellas CV. Reestablishment of occlusion through overlay removable partial dentures: a case report. Braz Dent J. 2012; 23:172-74. https://doi.org/10.1590/S0103-64402012000200014

11. Demir T, Kecik D, Cehreli ZC. Kenny-Caffey Syndrome: Oral Findings and 4-year Follow-up of Overlay Denture Therapy. J Dent Child. 2007;74:236-40.

12. Nosouhian S, Davoudi A, Derhami M. Posterior open occlusion management by registration of overlay removable partial denture: A clinical report. J Indian Prosthodont Soc. 2015;15:386-89 https://doi.org/10.4103/0972-4052.171822

13. Bloom DR, Padayachy JN. Increasing occlusal vertical dimension-why, when and how. Br Dent J. 2006;200:251-56 https://doi.org/10.1038/sj.bdj.4813305

14. Mehta SB, Banerji S, Millar BJ, Suarez-Feito JM. Current concepts on the management of tooth wear: part 1. Assessment, treatment planning and strategies for the prevention and the passive management of tooth wear. Br Dent J. 2012;212:17-27.

https://doi.org/10.1038/sj.bdj.2011.1099

15. Ganddini MR, Al-Mardini M, Graser GN, Almog D. Maxillary and mandibular overlay removable partial dentures for the restoration of worn teeth. J Prosthet Dent 2004;91:210-14. https://doi.org/10.1016/j.prosdent.2003.12.021

16. Beumer III J, Hamada MO, Lewis S. A prosthodontic overview. Int J Prosthodont. 1993;6:126-30 https://doi.org/10.1097/00008505-199312000-00009

17. Del Castillo R, LaMar F, Ercoli C. Maxillary and mandibular overlay removable partial dentures for the treatment of posterior openocclusal relationship: a clinical report. J Prosthet Dent. 2002;87: 587-92.

https://doi.org/10.1067/mpr.2002.125578 